\title{
Il Grande Oltre
}

di Giuliano Boccali

giuliano.boccali@unimi.it

La tematica centrale de $I l$ Grande Oltre è impostata immediatamente nell'Introduzione dall'Autore, Franco Chiereghin, formulando le domande alle quali egli si propone di rispondere: «Con quali dinamiche si è configurato il pensiero delle origini più remote della tradizione indoaria? In virtù di quali articolazioni il pensiero si è spinto a concepire l'Origine e, in essa, la propria origine in quanto pensiero? Attraverso quali forme espressive ha saputo differenziare il pensiero dell'Origine, di ciò che è "Primo senza un secondo", da ciò che ha un'esistenza "seconda e derivata?"» (p. 19). In precedenza (p. 13, in sede di Premessa) Chiereghin ha dichiarato che le proprie competenze «appartengono a un ambito... molto circoscritto, quello [appunto] dell'analisi delle dinamiche del pensiero». L'affermazione di grande misura e discrezione - requisiti questi di assoluto pregio, ai giorni nostri soprattutto - può suscitare un sorriso nel recensore se è un indologo, e forse non soltanto: nella prospettiva per esempio di alcuni pensatori buddhisti, infatti, l'ambito dichiarato da Chiereghin come "molto circoscritto" si identifica, né più né meno, con l'intero universo, esteriore e interiore, distinzione peraltro che non ha senso in quel contesto; la posizione non è troppo lontana, più di un millennio in anticipo, da quella dell'idealismo assoluto di Berkeley, e senza nemmeno avere bisogno di Dio.

In ogni modo, i riferimenti di cui Chiereghin intende valersi per rispondere ai quesiti posti sono le ricerche "di due grandi e celebri maestri» (p. 19: Yajñavalkya e Naciketas) delle Upanishad, i testi che a partire dal VII secolo a.C. circa segnano la svolta di interiorizzazione all'interno della spiritualità indoaria. Il discrimine fissato da questi testi è colto da Chiereghin con grande 
precisione: posto che «il cuore pulsante della spiritualità indoaria» (ibidem) è il sacrificio (yajña), le Upanishad ne trasferiscono con incalcolabili effetti la pratica dal culto esteriore «offerto da un sacrificante e officiato da sacerdoti» (ibidem) alla realizzazione interiore consistente - anticipiamo qui sommariamente - nell'offerta del proprio sforzo di superamento dei limiti fenomenici, per accedere all'identicazione della propria essenza eterna nel brahman, l'assoluto ineffabile e privo di qualità (nirguna), nonché all'affermazione della propria assoluta libertà nell'atto dissipativo del sacrificio. È così tratteggiato in maniera assai limpida lo scenario entro il quale «il pensiero [indiano originario] elabora le sue dinamiche più caratteristiche e determinanti» (ibidem) compendiate felicemente dall'Autore con il titolo del suo libro tratto da un'espressione dell'Atharva-veda (X, 7, 25) nella traduzione di R. Panikkar citata a p. 35, n. 32. Opportunamente, prima di entrare nell'indagine, così impostata, sulle ricerche di Yajñavalkya e Naciketas che operano una vasta e radicale rivoluzione del pensiero, Chiereghin ne esamina gli antefatti attraverso lo studio di tre inni del Rigveda fondamentali allo scopo di riconoscere «la trama concettuale che sta alla base dell'esperienza sacrificale» (p. 22). Si tratta degli inni Rigveda X,129, Rigveda X, 81 e Rigveda X, 90; i temi affrontati alla luce di questi testi sono le dinamiche del pensiero ravvisate all'Origine dai veggenti in: potenza della negazione, autoriferimento, ricorsività «che entrano a costituire le direttrici profonde su cui si viene costruendo la sapienza indoaria» (p. 33). Di seguito, l'Autore indaga il sacrificio integrale dell'Uno, "atto che schiude la forza originante del Principio» (p. 40); il sacrificio come «accrescimento» (p. 46 sgg.) del dio, come «autotrascendimento», che chiama l'essere umano al compito radicalmente nuovo della rinuncia incarnato nella condizione del "morto in vita" che rappresenterà, come è noto, l'ideale supremo di realizzazione spirituale dell'uomo nell'induismo ascetico.

L'indagine prosegue così approfondendo il tema del sacrificio considerato «paradigma perfetto dell'agire» (p. 54) dove, riprendendo in sede teorica la 
formula celeberrima rigvedica $\left(\operatorname{Rig}^{\circ} \mathrm{X}, 90,16\right)$ «con questo sacrificio gli dèi fecero un sacrificio al sacrificio» (yajñéna yajñám ayajanta devás, citata a p. 53), «il soggetto, il mezzo e il fine dell'azione sono il medesimo». Questa struttura identitaria, la cui essenza è tragica (p. 55), offre all'Autore l'opportunità di un confronto pertinente quanto fecondo con la praxis tragica in Aristotele.

Il tema del sacrificio deve necessariamente includere quello della morte, che Chiereghin introduce esaminando il mito della lotta fra Prajapati, il "Signore delle creature" e Mrityu, "Morte" (maschile in sanscrito) che parimenti ambiscono alla sovranità sui viventi e che appaiono forti di pari armamenti. A prevalere è il primo, grazie alla supremazia su Morte del pensiero capace, in virtù della sua potenza, di assimilare a sé il rivale senza esserne distrutto. La conclusione è raggiunta dall'Autore anche grazie all'originale paragone con il conflitto, nella ballata di Goethe, fra il cavaliere e il re degli Elfi (Erlkönig), dove il padre cerca invece di vincere la morte antagonista "naturalizzandola" anziché assimilarla e perciò soccombe nel duello estremo per la vita del figlio.

L'essenza del sacrificio è infine ravvisata da Chiereghin nell'interrogarsi sul secondo, enigmatico, momento del rito, quando «parte della vittima, di cui ci si potrebbe cibare, viene invece avviata alla sua distruzione pura e semplice col fuoco» (p. 68). Il gesto, che costituisce una «dissipazione assurda, definitiva e irrimediabile» (p. 72), ha "la regale magnificenza dell'assoluta libertà» (ibidem): mostra infatti la capacità da parte dell'uomo di svincolarsi "dalle strettoie della necessità" e di sapersi libero, affermando la propria indipendenza dalla sfera dei bisogni e dal circuito altrimenti fatale di bisognosoddisfazione e di indigenza-appagamento.

In sintesi, riprendendo e ampliando quanto già riepilogato poco sopra: «prima di affrontare le vicende umane e speculative di Yajñavalkya e di Naciketas» (p. 73), che rappresentano l'oggetto principale della ricerca di Chiereghin, «le dinamiche sviluppate dal pensiero [nelle] più antiche 
testimonianze dello hinduismo» (ibidem) sono costituite dall'autosacrificio del pensiero stesso, dal neti («non così non così)) che lo pone "al cospetto dell'Origine più antica» (ibidem), dall'esperienza dell'autoriferimento e da quella della ricorsività. Queste dinamiche fondanti si condensano e risolvono nella formula già ricordata del sacrificio, l'«azione perfetta», che «racchiude in sé il senso e il destino più alti e inoltrepassabili del divino e dell'umano" (p. 74). Chi scrive non solo condivide personalmente senza riserve questa conclusione di Chiereghin, ma ritiene - in quanto studioso dell'India tradizionale - che raramente sia stato colto in maniera altrettanto efficace e profonda il cuore della civiltà indiana e individuato l'orientamento del suo più alto destino interiore mettendone al tempo stesso acutamente in luce la portata sul piano del pensiero universale.

Questa valutazione sintetica - desidero qui anticipare - è da me riferita all'intero libro del filosofo padovano (nativo di Chioggia), di cui mi accingo a continuare la presentazione non senza un'autolimitazione di natura personale, che spero possa risultare tuttavia accettabile.

Interludio: è posizione attribuita ai grandi poeti in generale e - per quanto mi risulta da testimonianze personali - a Montale in particolare quella di limitarsi, se interrogati sul significato di una loro poesia, a invitare a rileggerla. Tutto il senso dei versi è da questi espresso, senza nessuna esclusione, aggiunta o necessità di interpretazione. Lo stesso vale per i compositori (o anche gli esecutori di talento) interrogati sul senso di un testo musicale... La convinzione aprirebbe abissi di ricerca, storica e teorica, sul piano dell'estetica: non è il caso nemmeno di sfiorarli qui, ammesso che chi scrive ne fosse capace, salvo (forse) entro i confini ristretti dell'estetica indiana.

La considerazione mi è tornata in mente leggendo e studiando il libro di Chiereghin: la ricchezza, la densità e l'accuratezza del suo argomentare, l'acume delle sue conclusioni sono tali a mio parere da non poter essere 
esposte sinteticamente, e men che meno spiegate con modalità interpretative. Consapevole di questo, ho cercato nella prima parte di questa recensione di procedere con una certa lentezza nel modo il più aderente possibile al processo della ricerca dell'Autore, così da offrirne almeno un'idea. A mio parere, però, proseguire in questa modalità richiederebbe da parte mia una sorta di riscrittura riassuntiva dell'opera; per cui a questo punto il suggerimento che posso dare di tutto cuore al Lettore... è quello di leggere (o ri-leggere) il libro, le cui convinzioni mi persuadono pienamente, salvo in aspetti, magari in qualche caso importanti, che però non incidono sulle acquisizioni rese disponibili dall'opera. Nel seguito del mio lavoro, perciò, mi limito non senza rammarico solamente a fornire qualche elemento sui due pensatori, Yajñavalkya e Naciketas, brahmani entrambi, studiati da Il Grande Oltre, nonché a esporre in sintesi le conclusioni raggiunte da Chiereghin circa $\mathrm{i}$ quesiti posti fin dal principio del libro o espansi e maturati nel corso dell'indagine.

Yajñavalkya è uno dei grandi protagonisti delle ricerche esposte dalla Brhadaranyaka Upanishad, come già lo era stato dello Shatapathabrahmana; il riferimento ai suoi interventi si trova a p. 77 , n. 126. La ricchezza dei temi da lui affrontati è straordinaria: la potenza della "parola" (vac) e della sacra sillaba om in particolare, le vie d'accesso al Brahman, il fondamento dell'"essere-caro", la morte e la trasfigurazione del rapporto amoroso, il tempo, l'inoggettivabilità del Principio e le vie d'accesso all'Origine sono a mo' di esempio solo alcuni fra gli argomenti dell'indagine che occupa i 5 capitoli della Parte II. Un punto di forza del percorso di Chiereghin sta nell'avere inquadrato le tenzoni dialogiche con i suoi interlocutori-rivali nella vicenda personale di Yajñavalkya, lumeggiando lo stile di vita del Maestro che ne distingue la personalità e che si evolve impregnandosi dell'evoluzione del suo pensiero progressivamente e sintonicamente. L'apice della formidabile ricerca è rappresentato (pp. 220-225) da $\mathrm{Brhad}^{\circ} \mathrm{V}, 1$, dove «l'inesauribilità della fonte 
primigenia fa sì che anche quando sia stato preso il pieno dal pieno, la pienezza originaria rimanga intatta» (p. 221); il che può essere interpretato adeguatamente solo in relazione con il sacrificio, unico atto in grado di collegare le due pienezze. L’indagine sul vertiginoso pensiero di Yajñavalkya si conclude mettendo in luce le conseguenze pratiche della posizione teoretica testé accennata, che consistono ( $\mathrm{Brhad}^{\circ} \mathrm{V}, 2$, analisi alle pp. $223 \mathrm{sgg}$.) negli imperativi etici di "dominarsi", "donare" "avere compassione". Sottolineo qui l'interesse delle riflessioni dedicate da Chiereghin a questi tre aspetti aggiungendo che - credo non a caso - essi coincidono con tre dei quattro principi costitutivi dell'induismo, cioè (per denominarlo secondo la sua stessa originale denominazione) del sanatana dharma, la "eterna legge (sacra)": questi sono infatti "autodominio", "generosità", "non-violenza"; il quarto è sat o satya, l'“essere", che coincide per la concezione indiana con il "vero"; è superfluo rilevare che proprio questo principio è stato sempre al centro della ricerca del grande Maestro upanishadico la quale si pone così ante litteram la religione hindu è ancora in fieri, all'epoca - come una grandiosa meditazione sull'ontologia e sui fondamenti etici che contraddistinguono l'induismo.

Analoga è la prospettiva di Chiereghin riguardo a Naciketas, figura «per alcuni aspetti complementare a quella di Yajñavalkya» (p. 231) sia per l'età è un fanciullo o poco più - sia per la personalità che appare già completamente formata e caratterizzata dall'amore filiale e da un'inestinguibile e pura sete di conoscenza. Questa sarà misurata nel memorabile dialogo con Yama, il dio della Morte, prova di iniziazione che costituisce la breve e straordinaria Katha Upanishad, molto più recente della $\mathrm{Brhad}^{\circ}$, forse risalente al IV-III secolo a. C. (Parte III, capp. 1-3). Il fanciullo-brahmano viene inviato dal padre a Yama - molto significative le riflessioni di Chiereghin (pp. 230-237) a proposito del singolare invio - e il giovane pazientemente deve attendere l'incontro per tre giorni, trascorrendoli in solitudine e digiuno: la Morte infatti è in viaggio, molto e in molti luoghi impegnata, come si sa... 
Per compensarlo dell'attesa, e per onorarlo in quanto brahmano, al proprio ritorno Yama offre a Naciketas di esaudire tre sue richieste: alla terza, ecco la prova più ardua! Morte lusinga infatti ripetutamente il giovane, suggerendogli di domandare ogni sorta di bene terreno; ma Naciketas è irremovibile: "danze, canti, carrozze, fanciulle, ricchezze e lunga vita", che fine farà tutto questo quando - insinua non senza arguzia - si sarà (nel suo caso per la seconda volta) al cospetto di Morte? Egli perciò «vuole "solo" che il suo pensiero sia condotto a penetrare» nel mistero di «ciò che succede nel grande passaggio» dalla vita alla morte. Le pagine di Chiereghin al riguardo, sulla scorta del testo upanishadico, sono davvero consistenti e le conclusioni riecheggiano sviluppandole in maniera assai profonda $i$ quesiti dell'impostazione sopra prospettata. L'orizzonte del desiderio è infatti superato, anzi dissolto nell'evidenza raggiunta dal fanciullo-brahmano: «il fuoco permanente è quello che arde nell'atto di rinuncia» analogamente alla «rinuncia... a una parte della vittima, bruciata e non consumata nel banchetto sacrificale» (p. 247). Il segreto della Morte consiste, come per il sole, nel suo duplice volto: la dissoluzione del mondo impermanente e finito, oltre che «insidiato da dualismi e disperso nella molteplicità» e al tempo stesso l'accesso a Brahman e «la via all'immortalità» (p. 251 ss.) Lo sviluppo di questa conclusione approda ad affermare lo «instaurarsi di un vera e propria "trascendenza interiore" in colui che "si lascia scegliere"... dall'aspetto solare con cui la Morte si rivolge al regno dell'immortalità» (p. 255) o, in altre parole, da Atman-Brahman. Si riflette in questa conclusione di Chiereghin la convinzione filosofica e spirituale, del tutto condivisa da chi scrive (e non estranea, credo, in altro contesto al De Anima di Aristotele), che l'irruzione del divino, del trascendente incondizionatamente libero, possa essere favorita, preparata, attesa, ma non mai causata: «suprema forma di attività... è [infatti ] il "lasciar essere" l'opera del dio... La dinamica ontologica che così viene allo scoperto mostra che nulla è più individualmente proprio a ciascuno di ciò che lo trascende per costituirlo nel suo essere più autentico...» (p. 255). 
Altro punto forte nell'indagine sulla vicenda di Naciketas nella Katha Upanishad è quello della «molteplicità redenta dall'amore» - come emblematicamente recita il titolo dell'ultimo capitolo della Parte III che in maniera penetrante si diffonde sulla possibilità di percepire l'Assoluto unicamente dicendo "[Esso] è [...] punto di immedesimazione e di assimilazione del cercante al cercato, del finito all'infinito, dello jivatman col Brahman» (p. 269). E punto quindi di accesso del ricercatore, già in vita e dopo la morte, all'immortalità del riassorbimento nell'Assoluto, nel Brahman. Tale unica possibilità è messa a confronto e al tempo stesso opportunamente differenziata da Chiereghin con «'insegnamento della dea a Parmenide nel mondo greco» (ibidem). Poco oltre la trattazione vera e propria dell'Autore si conclude con parole che idealmente la ricongiungono al principio del libro (come pure al Principio indagato dai Veda): «Ma anche qui occorre tenere ferma la natura ancipite di quest'esperienza di immedesimazione racchiusa nell'“è". Essa è dono e sottrazione ad un tempo. Nell'atto in cui nell'“è" il Principio offre l'attestazione più potente della sua presenza, esso si ritrae nell'inaccessibilità, perché niente altro è possibile "dire" di Lui. L“è" come incessante dono della propria pienezza e quale ininterrotto attingere il pieno dal pieno, lascia il pieno irrappresentabile nella sua inesauribile sovrabbondanza» (p. 273).

Se una valutazione molto sintetica è lecita (anche se del tutto carente) per la straordinaria costruzione di un'opera densa, puntualmente argomentata e appassionata insieme come quella di Chiereghin, si vorrebbe dire che essa mantiene pienamente l'assunto espresso dal suo titolo "Il Grande Oltre" e che l'indagine ritorna - dopo sviluppi articolati, vertiginosi e assolutamente convincenti - all'esperienza delle origini espressa, fra l'altro, dall'inno a Purusha (Rgveda X, 90): il grande Essere dell'origine è più grande della sua grandezza (str. 3); l'Essere è traboccamento, sovrabbondanza inesauribile (e sarebbe meglio dire non decrementabile) coincidente, come già si è accennato 
e come la stessa mitica immagine della cosmica oblazione costituita da Purusha dimostra, con il sacrificio totale di sé.

Una riflessione finale da parte mia, in parte accessoria (ma non irrilevante) e in parte sostanziale:

Va davvero elogiata la cura grande che Il Grande Oltre pone a tutto quello che concerne la nomenclatura in sanscrito e la trascrizione dei termini, che rigorosamente segue l'uso scientifico; un requisito davvero rarissimo nelle opere che trattano di argomenti indiani. Ma rarissima soprattutto è la capacità di Chiereghin di presentare e interpretare le tematiche svolte dai testi e dai pensatori indiani considerati come tematiche filosofiche tout court, di respiro e significato generale, non legate necessariamente alla cultura e all'epoca determinata dove sono state elaborate, capaci di sollecitare e interrogare ciascuno qualunque sia il suo orizzonte culturale.

Franco Chiereghin, Il Grande Oltre. Il cammino di pensiero aperto da Yajñavalkya e da Naciketas nelle Upanishad, "Rationes", Padova University Press, Padova 2019, pp. 309, € 14,00. 